\title{
Effects of Propranolol on Neurodevelopmental Outcomes in Patients with Infantile Hemangioma: A Case-Control Study
}

\author{
Chuan Wang, ${ }^{1}$ Qi Wang, ${ }^{1}$ Bo Xiang, ${ }^{1}$ Siyuan Chen, ${ }^{2}$ Fei Xiong, ${ }^{3}$ and Yi Ji $\mathbb{D}{ }^{1}$ \\ ${ }^{1}$ Division of Oncology, Department of Pediatric Surgery, West China Hospital of Sichuan University, Chengdu 610041, China \\ ${ }^{2}$ Pediatric Intensive Care Unit, Department of Critical Care Medicine, West China Hospital of Sichuan University, \\ Chengdu 610041, China \\ ${ }^{3}$ Department of Child Health, West China Second University Hospital, Sichuan University, Chengdu 610041, China
}

Correspondence should be addressed to Yi Ji; jijiyuanyuan@163.com

Received 9 September 2017; Revised 28 December 2017; Accepted 4 January 2018; Published 25 February 2018

Academic Editor: Jean Kanitakis

Copyright (C) 2018 Chuan Wang et al. This is an open access article distributed under the Creative Commons Attribution License, which permits unrestricted use, distribution, and reproduction in any medium, provided the original work is properly cited.

Background. The aim of this study was to examine whether oral propranolol has any effect on neurodevelopment outcomes in young children with problematic infantile hemangiomas (IHs). Methods. Thirty-six children with a diagnosis of problematic IH who were treated with oral propranolol were compared with 34 healthy children with no history of propranolol therapy. Patients received propranolol therapy for at least 3 months. Gesell developmental schedules (GDS) were used to evaluate neurodevelopment outcomes in the two groups. The scores of each GDS domain were compared between the two groups. Results. There were no significant differences in developmental quotient (DQ) values for any of the five domains between the patients and healthy controls $(P<0.05)$. Multiple stepwise regression analyses showed that none of the domains in the control group were influenced by the children's gender or age $(P<0.05)$. In addition, we found that gender, age at the initiation of therapy, age at the time of the neurodevelopment test, and treatment duration had no effect on any domain of the GDS in the patient group $(P>0.05)$. Conclusion. Propranolol has no obvious effect on neurodevelopmental outcomes in children. Early treatment and treatment duration had no negative effect on central nervous system (CNS) development.

\section{Introduction}

Infantile hemangioma (IH) is the most common benign tumor in children. Most IHs are small, do not cause any complications, and involute naturally without treatment. However, approximately $10-15 \%$ of IHs can lead to compromised function and permanent disfigurement or are lifethreatening. In certain cases, timely intervention is crucial for preventing potential sequelae $[1,2]$. Currently, propranolol is the first-line treatment for IHs due to its excellent efficacy [3]. However, adverse events are common, including sleep disturbance, agitation, acrocyanosis, and gastrointestinal symptoms [4]. Some serious adverse events, including symptomatic hypotension, hypoglycemia, and bradycardia, have also been reported [3].

Propranolol is a lipophilic nonselective $\beta$-adrenergic receptor ( $\beta$-AR) blocker and has the ability to easily penetrate the blood-brain barrier [5]. It is well established that noradrenaline neurotransmitters play an important role in the early development of the central nervous system (CNS) [6]. Both agonists and antagonists of $\beta$-ARs act antithetically via the same intracellular pathways [7]. The use of propranolol in the treatment of IHs is usually initiated in infancy, which is a critical time for CNS development. These issues raise questions regarding the assumption that propranolol may have the capacity to exert effects on CNS development in young infants [8].

Interestingly, although clinical data in children are rare, evidence for the effects of propranolol on CNS function in adults is plentiful, albeit controversial [8]. In this regard, studies demonstrated that propranolol could impair memory, psychomotor functions, and mood in adults $[6,8]$. Mihov et al. found that propranolol had an obvious effect on the facilitation of declarative learning by social-emotional feedback [9]. Remarkably, sleep disturbance is one of the most common adverse effects of propranolol in infants [10]. There 
is direct evidence that acute sleep restriction has a negative impact on emotion responses in children [11].

Unfortunately, although propranolol is widely used to treat IHs, little is known regarding the impact of propranolol on CNS development in children. Clinically, the initiation of propranolol treatment for cosmetic IHs is a difficult choice to make. Therefore, we conducted this study to assess the impact of propranolol on CNS function in young children by using Gesell developmental schedules (GDS) and identifying related risk factors of CNS function (if they exist), with the aim of providing meaningful data regarding the safety of propranolol therapy in children.

\section{Methods}

This study was approved by the Ethics Committee of West China Hospital of Sichuan University and West China Second University Hospital of Sichuan University. All procedures followed the research protocols that were approved by West China Hospital of Sichuan University and West China Second University Hospital of Sichuan University and were conducted according to the Declaration of Helsinki.

2.1. Patients and Health Children. All patients with IH were recruited at the Department of Pediatric Surgery, West China Hospital of Sichuan University, from January 2015 to December 2015. The inclusion criteria were as follows: (1) patients were aged $<12$ months; (2) patients had received propranolol therapy due to problematic IHs, and the duration of treatment was $\geq 3$ months; and (3) patients did not have any known risk factor for developmental delay or growth restriction. The exclusion criteria were the following: (1) patients had received or were currently receiving other treatments or any comedication for IHs; (2) the duration of propranolol treatment was <3 months; and (3) patients have PHACE syndrome (posterior fossa malformations, hemangiomas, arterial malformations, coarctation of the aorta and other cardiac defects, and eye anomalies). Age- and gender-matched control subjects were healthy children. They were recruited from the Department of Child Health, West China Second University Hospital of Sichuan University. Written informed consent was obtained from each child's parents.

2.2. Propranolol Treatment. The patients' parents were informed that propranolol was prescribed for the treatment of $\mathrm{IH}$, and they provided written, informed consent for treatment. Propranolol was initiated at a dose of $1.0 \mathrm{mg} / \mathrm{kg} /$ day divided 3 times daily for 1 week, and the dose was then increased to $2.0 \mathrm{mg} / \mathrm{kg} /$ day in 3 daily doses beginning in week 2 . The dosage of propranolol was adjusted for weight $(2 \mathrm{mg} / \mathrm{kg}$ per day). The duration of propranolol use was noted.

2.3. Instruments. GDS, developed by Gesell, were designed to evaluate broad CNS development. This instrument is widely used due to its excellent reliability and validity [12]. GDS provided a neurodevelopmental profile in five domains (adaptive, gross motor, fine motor, language, and personal/social) [12]. The neurodevelopmental status was measured by the development quotient (DQ) of the five domains. The DQ was derived by dividing the age-equivalent level of each domain according to the child's chronological age at the time of the neurodevelopmental assessment [13]. A higher DQ indicates better neurodevelopment. Considering that prematurity is an important risk factor for IH [14], GDS may be better for the assessment of neurodevelopmental status than other instruments. GDS was conducted by specially trained pediatric physicians and nurses in West China Second University Hospital of Sichuan University.

2.4. Statistical Analysis. All analyses were conducted by using SPSS 22.0 for Windows (SPSS Inc., Chicago, IL, USA). An independent samples $t$-test was used to analyze the continuous variables, and chi-square $(\chi 2)$ tests were used to compare qualitative variables. Multiple stepwise regression analyses were used to identify the main factors influencing GDS. Statistical significance was set at $P<0.05$.

\section{Results}

Thirty-six patients with IHs were recruited in this study. And thirty-four healthy children with a mean age of 12.59 months were enrolled in the healthy control group. Demographic characteristics of two groups were listed in Table 1 . There were no significant differences in demographic characteristics between the two groups $(P>0.05)$.

DQ values of each domain in the two groups are shown in Table 2. We found no significant difference between corresponding DQ values in the five domains in the two groups $(P>0.05)$.

The outcomes of multiple stepwise regression analyses of DQ values of each domain in the healthy control group and the patient group are listed in Tables 3 and 4, respectively. In the control group, age and gender did not have a significant effect on DQ values in any of the GDS domains $(P>0.05)$. In the patient group, we found that gender, age at the initiation of therapy, age at the time of the neurodevelopment test, and treatment duration had no effect on any domain of the GDS $(P>0.05)$.

\section{Discussion}

Propranolol has been widely used to treat IHs since Léautélabrèze et al. serendipitously discovered the dramatic effects of propranolol in inducing an accelerated involution of problematic IHs [15]. However, although propranolol is efficacious, there are theoretical concerns regarding the potential significant neurodevelopmental or cognitive side effects of propranolol in children [16].

Noradrenaline neurotransmitters in the CNS are mainly released from the locus coeruleus and are strongly associated with the functions of the amygdaloid nucleus and the hippocampus [17], both of which are involved in emotion experience [18-20]. The high liposolubility of propranolol can facilitate the passage of propranolol across the bloodbrain barrier. Thus, propranolol has been used to relieve anxiety symptoms and prevent posttraumatic stress disorder 
TABLE 1: Demographic characteristics of the patient group and the healthy group.

\begin{tabular}{|c|c|c|c|}
\hline Variable & Patient group & Control group & $P$ values \\
\hline Gender* & & & 0.663 \\
\hline Male & $13(36.1)$ & $14(41.2)$ & \\
\hline Female & $23(63.9)$ & $20(58.8)$ & \\
\hline \multicolumn{4}{|l|}{ Gestational age* $^{*}$} \\
\hline Term born ( $\geq 37$ weeks) & $30(83.3)$ & $29(85.3)$ & 0.822 \\
\hline Born prematurely (<37 weeks) & $6(16.7)$ & $5(14.7)$ & \\
\hline Age at neurodevelopment test $(\mathrm{m})$ & $12.27 \pm 1.56$ & $12.59 \pm 2.06$ & 0.478 \\
\hline
\end{tabular}

${ }^{*}$ Values are presented as the number (percentage). Values are presented as the mean \pm standard deviation.

TABLE 2: Scores of each domain in the patient group and the healthy group* .

\begin{tabular}{lccc}
\hline Domain of GDS & Patient group (mean \pm SD) & Control group (mean \pm SD) & $P$ values \\
\hline Adaption & $94.81 \pm 10.01$ & $96.57 \pm 16.21$ & 0.584 \\
Gross motor & $98.20 \pm 11.41$ & $95.39 \pm 9.40$ & 0.365 \\
Fine motor & $101.98 \pm 11.81$ & $102.20 \pm 15.28$ & 0.945 \\
Language functioning & $88.53 \pm 12.05$ & $88.01 \pm 15.13$ & 0.872 \\
Personal social functioning & $98.11 \pm 17.48$ & $92.38 \pm 9.19$ & 0.940 \\
\hline
\end{tabular}

${ }^{*}$ GSD: Gesell developmental schedules; SD: standard deviation.

TABLE 3: Factors influencing the scores of each domain in the healthy group* ${ }^{*}$

\begin{tabular}{|c|c|c|c|c|}
\hline \multirow{2}{*}{ Variable } & \multicolumn{2}{|c|}{ Gender } & \multicolumn{2}{|c|}{ Age } \\
\hline & $\beta$ values (95\% CI) & $P$ values & $\beta$ values (95\% CI) & $P$ values \\
\hline Adaption & $-0.008(-10.703-10.263)$ & 0.966 & $0.144(-1.531-3.549)$ & 0.424 \\
\hline Gross motor & $-0.124(-8.866-4.212)$ & 0.473 & $0.301(-0.214-2.956)$ & 0.087 \\
\hline Fine motor & $-0.161(-15.967-6.092)$ & 0.368 & $-0.082(-3.279-2.066)$ & 0.647 \\
\hline Language functioning & $-0.075(-13.198-8.651)$ & 0.674 & $-0.162(-3.834-1.460)$ & 0.367 \\
\hline Personal social functioning & $-0.179(-9.862-3.268)$ & 0.314 & $-0.139(-2.210-0.971)$ & 0.443 \\
\hline
\end{tabular}

${ }^{*}$ Multiple stepwise regression analyses were used to determine the main risk factors; CI: confidence interval.

$[21,22]$. Stoschitzky et al. demonstrated that propranolol was able to block the 5-hydroxytryptamine receptor, resulting in decreased production of melatonin and influencing CNS function [23]. Clinically, children who receive propranolol therapy may be at a higher risk of CNS-related adverse events (e.g., sleep disturbance, agitation, and somnolence) [10]. Therefore, whether propranolol has a negative impact on CNS function in children is a significant concern. Clinicians may face a dilemma in determining whether to treat IHs with propranolol for a cosmetic reason.

The accurate measurement of CNS function in normal children is sometimes difficult. GDS could be used to broadly assess the neurodevelopmental status in children; additionally, they provide a reliable measure of development in this age group and yield separate scores for mental and motor development $[24,25]$. In the present study, we used GDS to determine that propranolol therapy had no negative impact on neurodevelopmental outcomes in young children with IHs.

Our study showed that oral propranolol at a dose of $2.0 \mathrm{mg} / \mathrm{kg} /$ day did not impair early fine or gross motor development in young children with IHs. These findings were consistent with the serial studies by Moyakine et al., which demonstrated that propranolol neither negatively influenced fine or gross motor function nor affected psychomotor development in patients with IH [26-28]. Remarkably, our study provides further evidence that propranolol therapy for a mean duration of 9.40 months did not impair adaption and personal social function in young children. Notably, the age at the initiation of propranolol treatment in our patients was in the first year of life, and this period is the most sensitive period for the development of language function [29]. We noted evidence for the equivalence between patients treated with propranolol and healthy controls in terms of language function domain. Our study provided valuable results indicating that propranolol had no negative impact on the development of language function.

As mentioned above, propranolol has an impact on the functions of the amygdaloid nucleus and the hippocampus. These two domains are also involved in memory function [30]. Therefore, concern rises continuously regarding the possible effects of propranolol on memory function. Cahill and Van Stegeren substantiated that the use of propranolol for a short time had a negative impact on the memory in adult [31]. However, CNS has a strong plasticity in infancy. Theoretically, alternative pathway may compensate the memory function 


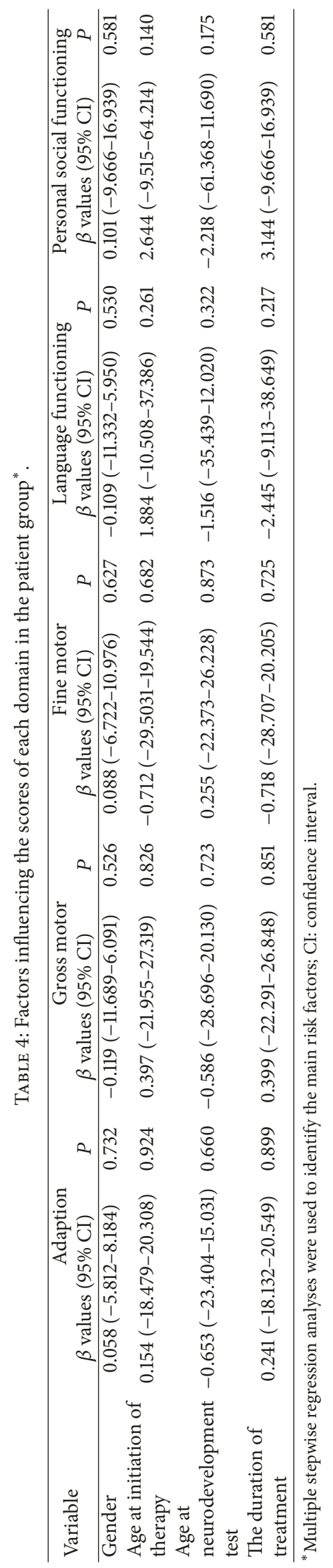


for a long-term use of propranolol. Unfortunately, previous studies failed in clarifying the puzzle [10]. In our study, the GDS is also unable to test the impact of propranolol on memory function. Therefore, question still exists as whether long-term use of propranolol has a negative effect on memory function in infancy. Further studies are needed to elucidate this question.

Recently, studies have revealed that the rebound or relapse of IHs occurred more frequently in patients who completed propranolol treatment before 9 months of life [32]. Usually, the duration of propranolol treatment was longer than 6 months [33]. In the present study, the mean duration of propranolol therapy was 9.40 months. Thus, the majority of our patients received propranolol treatment with a duration that may cover the entire proliferative phase. Although several studies have shown that propranolol had a significantly negative impact on CNS function, the duration of oral propranolol was short $[9,30,34]$. Otherwise, it is conceivable that CNS function was influenced by propranolol in the short term, but this function may be compensated by brain plasticity during long-term treatment.

We found that the age at the initiation of therapy had no effect on CNS development. This finding is exciting and suggests that the CNS risks associated with the use of propranolol in young infants may be lower than we initially feared. Previously, Chang et al. demonstrated that the largest increase in IH tumor size occurred in the first 3 months of life, and by 5 months of age, both segmental and localized IHs had reached $80 \%$ of their final size [35]. Some IHs are associated with a high risk of functional or cosmetically critical changes even in the early proliferative phase. Although propranolol treatment is effective in both arresting growth and causing involution, irreversible sequelae may have already occurred. These observations, together with the work presented here, promote the recommendation that, in patients with severe IHs, oral propranolol should be administered as early as possible to avoid potential complications $[36,37]$.

The limitations of this study include its resultant small cohort sizes. In addition, some CNS functions cannot be accurately measured until the age of 6-7 years. There is insufficient data, at our institutions and in the literature, in reporting long-term CNS function in order to ensure the safety of propranolol in the treatment of IHs.

\section{Conclusion}

In conclusion, we demonstrated satisfactory neurodevelopmental outcomes in IH patients treated with propranolol. Although long-term follow-up data are lacking, our current data demonstrated that propranolol does not produce neurodevelopmental delays in young children for short-term use. In addition, we revealed that the duration of propranolol therapy did not increase neurodevelopmental risk. Early treatment with propranolol had no negative effect on CNS function.

\section{Conflicts of Interest}

The authors declare that they have no conflicts of interest.

\section{Authors' Contributions}

Chuan Wang and Qi Wang contributed equally to this work.

\section{Acknowledgments}

This work was supported by grants from the National Natural Science Foundation of China (81401606 and 81400862) and the Science Foundation for Excellent Youth Scholars of Sichuan University (2015SU04A15). The authors would like to thank their patients' parents for their cooperation and support and for providing their consent regarding the publication of this manuscript.

\section{References}

[1] P. H. Hoeger, J. I. Harper, E. Baselga et al., "Treatment of infantile haemangiomas: recommendations of a European expert group," European Journal of Pediatrics, vol. 174, no. 7, pp. 855865, 2015.

[2] Y. Ji, S. Chen, B. Xiang et al., "Clinical features and management of multifocal hepatic hemangiomas in children: a retrospective study," Scientific Reports, vol. 6, Article ID 31744, 2016.

[3] A. L. Marqueling, V. Oza, I. J. Frieden, and K. B. Puttgen, "Propranolol and infantile hemangiomas four years later: a systematic review," Pediatric Dermatology, vol. 30, no. 2, pp. 182191, 2013.

[4] C. E. Cheng and S. F. Friedlander, "Infantile hemangiomas, complications and treatments," Seminars in Cutaneous Medicine and Surgery, vol. 35, no. 3, pp. 108-116, 2016.

[5] A. Wood, K. Carr, R. Vestal, S. Belcher, G. Wilkinson, and D. Shand, "Direct measurement of propranolol bioavailability during accumulation to steady-state.," British Journal of Clinical Pharmacology, vol. 6, no. 4, pp. 345-350, 1978.

[6] B. A Bryan, "Reconsidering the use of propranolol in the treatment of cosmetic infantile hemangiomas," Angiology: Open Access, vol. 01, no. 01, 2013.

[7] Y. Ji, S. Chen, K. Li, X. Xiao, S. Zheng, and T. Xu, "The role of $\beta$-adrenergic receptor signaling in the proliferation of hemangioma-derived endothelial cells," Cell Division, vol. 8, no. 1, 2013, article no. 1 .

[8] A. Langley and E. Pope, "Propranolol and central nervous system function: potential implications for paediatric patients with infantile haemangiomas," British Journal of Dermatology, vol. 172, no. 1, pp. 13-23, 2015.

[9] Y. Mihov, S. Mayer, F. Musshoff, W. Maier, K. M. Kendrick, and R. Hurlemann, "Facilitation of learning by social-emotional feedback in humans is beta-noradrenergic-dependent," Neuropsychologia, vol. 48, no. 10, pp. 3168-3172, 2010.

[10] C. Léaute-Labrèze, O. Boccara, C. Degrugillier-Chopinet et al., "Safety of oral propranolol for the treatment of infantile hemangioma: a systematic review," Pediatrics, vol. 138, no. 4, Article ID e20160353, 2016.

[11] R. H. Berger, A. L. Miller, R. Seifer, S. R. Cares, and M. K. Lebourgeois, "Acute sleep restriction effects on emotion responses in 30- to 36-month-old children," Journal of Sleep Research, vol. 21, no. 3, pp. 235-246, 2012.

[12] R. Dror, G. Malinger, L. Ben-Sira, D. Lev, C. G. Pick, and T. Lerman-Sagie, "Developmental outcome of children with enlargement of the Cisterna Magna identified in Utero," Journal of Child Neurology, vol. 24, no. 12, pp. 1486-1492, 2009. 
[13] J. Meinzen-Derr, S. Wiley, S. Grether, and D. I. Choo, "Children with cochlear implants and developmental disabilities: a language skills study with developmentally matched hearing peers," Research in Developmental Disabilities, vol. 32, no. 2, pp. 757-767, 2011.

[14] A. Munden, R. Butschek, W. L. Tom et al., "Prospective study of infantile haemangiomas: incidence, clinical characteristics and association with placental anomalies," British Journal of Dermatology, vol. 170, no. 4, pp. 907-913, 2014.

[15] P. Cuccaro, A. Rapacciuolo, R. Vitiello et al., "Propranolol for severe hemangioma of infancy," Journal of Cardiovascular Medicine, vol. 12, no. 1, p. 5, 2011.

[16] P. H. Hoeger, "Propranolol for infantile haemangiomas: certain chances, potential risks," British Journal of Dermatology, vol. 172, no. 1, pp. 3-4, 2015.

[17] K. Itoi, "Ablation of the central noradrenergic neurons for unraveling their roles in stress and anxiety," Annals of the New York Academy of Sciences, vol. 1129, pp. 47-54, 2008.

[18] L. Cahill, R. Babinsky, H. J. Markowitsch, and J. L. Mc Gaugh, "The amygdala and emotional memory," Nature, vol. 377, no. 6547, pp. 295-296, 1995.

[19] L. Cahill, B. Prins, M. Weber, and J. L. McGaugh, “ $\beta$-Adrenergic activation and memory for emotional events," Nature, vol. 371, no. 6499, pp. 702-704, 1994.

[20] B. A. Strange and R. J. Dolan, “ $\beta$-Adrenergic modulation of emotional memory-evoked human amygdala and hippocampal responses," Proceedings of the National Acadamy of Sciences of the United States of America, vol. 101, no. 31, pp. 11454-11458, 2004.

[21] P. E. Hayes and S. C. Schulz, "Beta-blockers in anxiety disorders," Journal of Affective Disorders, vol. 13, no. 2, pp. 119-130, 1987.

[22] R. K. Pitman, K. M. Sanders, R. M. Zusman et al., "Pilot study of secondary prevention of posttraumatic stress disorder with propranolol," Biological Psychiatry, vol. 51, no. 2, pp. 189-192, 2002.

[23] K. Stoschitzky, A. Sakotnik, P. Lercher et al., "Influence of betablockers on melatonin release," European Journal of Clinical Pharmacology, vol. 55, no. 2, pp. 111-115, 1999.

[24] L. Hudon, J. Moise K.J., S. E. Hegemier et al., "Long-term neurodevelopmental outcome after intrauterine transfusion for the treatment of fetal hemolytic disease," American Journal of Obstetrics \& Gynecology, vol. 179, no. 4, pp. 858-863, 1998.

[25] W. D. Williamson, G. S. Wilson, M. H. Lifschitz, and S. A. Thurber, "Nonhandicapped very-low-birth-weight infants at one year of age: developmental profile," Pediatrics, vol. 85, no. 3, pp. 405-410, 1990.

[26] A. V. Moyakine, D. J. J. Hermans, J. Fuijkschot, and C. J. M. Van Der Vleuten, "Propranolol treatment of infantile hemangiomas does not negatively affect psychomotor development," Journal of the American Academy of Dermatology, vol. 73, no. 2, pp. 341342, 2015.

[27] A. V. Moyakine, J. M. Kerstjens, S. Spillekom-Van Koulil, and C. J. M. Van Der Vleuten, "Propranolol treatment of infantile hemangioma $(\mathrm{IH})$ is not associated with developmental risk or growth impairment at age 4 years," Journal of the American Academy of Dermatology, vol. 75, no. 1, pp. 59-63.el, 2016.

[28] A. V. Moyakine, S. Spillekom-van Koulil, and C. J. M. van der Vleuten, "Propranolol treatment of infantile hemangioma is not associated with psychological problems at 7 years of age," Journal of the American Academy of Dermatology, vol. 77, no. 1, pp. 105-108, 2017.
[29] V. Colletti, M. Carner, V. Miorelli, M. Guida, L. Colletti, and F. G. Fiorino, "Cochlear implantation at under 12 months: report on 10 patients," The Laryngoscope, vol. 115, no. 3, pp. 445-449, 2005.

[30] R. Hurlemann, H. Walter, A. K. Rehme et al., "Human amygdala reactivity is diminished by the $\beta$-noradrenergic antagonist propranolol," Psychological Medicine, vol. 40, no. 11, pp. 18391848, 2010.

[31] L. Cahill and A. Van Stegeren, "Sex-related impairment of memory for emotional events with $\beta$-adrenergic blockade," Neurobiology of Learning and Memory, vol. 79, no. 1, pp. 81-88, 2003.

[32] S. D. Shah, E. Baselga, C. McCuaig et al., "Rebound growth of infantile hemangiomas after propranolol therapy," Pediatrics, vol. 137, no. 4, Article ID e20151754, 2016.

[33] C. Léauté-Labrèze, J. I. Harper, and P. H. Hoeger, "Infantile haemangioma," The Lancet, vol. 390, no. 10089, pp. 85-94, 2017.

[34] J. Przybyslawski and S. J. Sara, "Reconsolidation of memory after its reactivation," Behavioural Brain Research, vol. 84, no. 1-2, pp. 241-246, 1997.

[35] L. C. Chang, A. N. Haggstrom, B. A. Drolet et al., "Growth characteristics of infantile hemangiomas: implications for management," Pediatrics, vol. 122, no. 2, pp. 360-367, 2008.

[36] Y. Ji, S. Chen, C. Xu, L. Li, and B. Xiang, “The use of propranolol in the treatment of infantile haemangiomas: an update on potential mechanisms of action," British Journal of Dermatology, vol. 172, no. 1, pp. 24-32, 2015.

[37] Y. Ji, S. Chen, B. Xiang, Y. Yang, and L. Qiu, "Safety and tolerance of propranolol in neonates with severe infantile hemangiomas: a prospective study," Scientific Reports, vol. 7, no. 1, article no. 1503, 2017. 


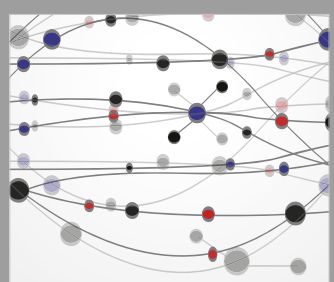

The Scientific World Journal
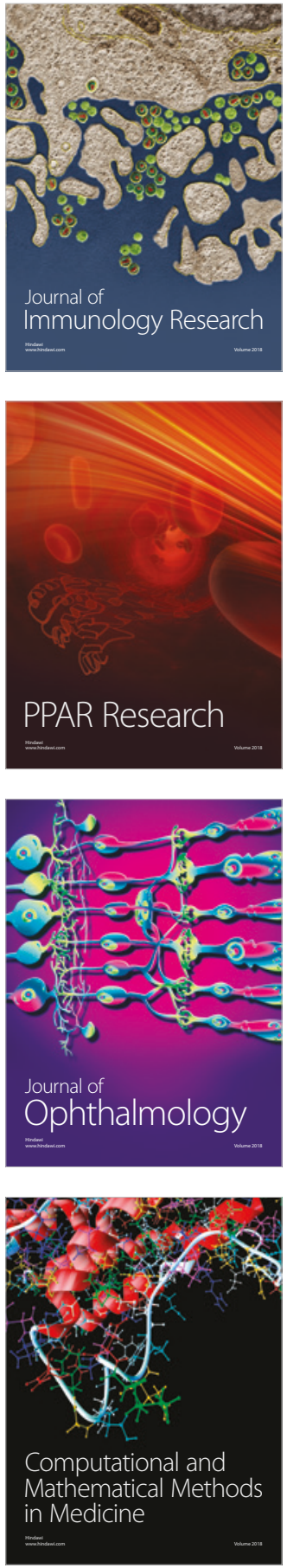

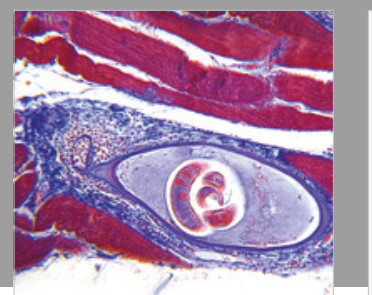

Gastroenterology Research and Practice

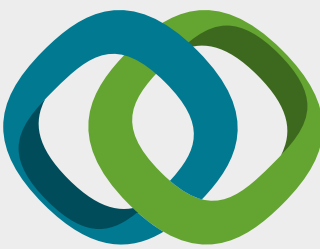

\section{Hindawi}

Submit your manuscripts at

www.hindawi.com
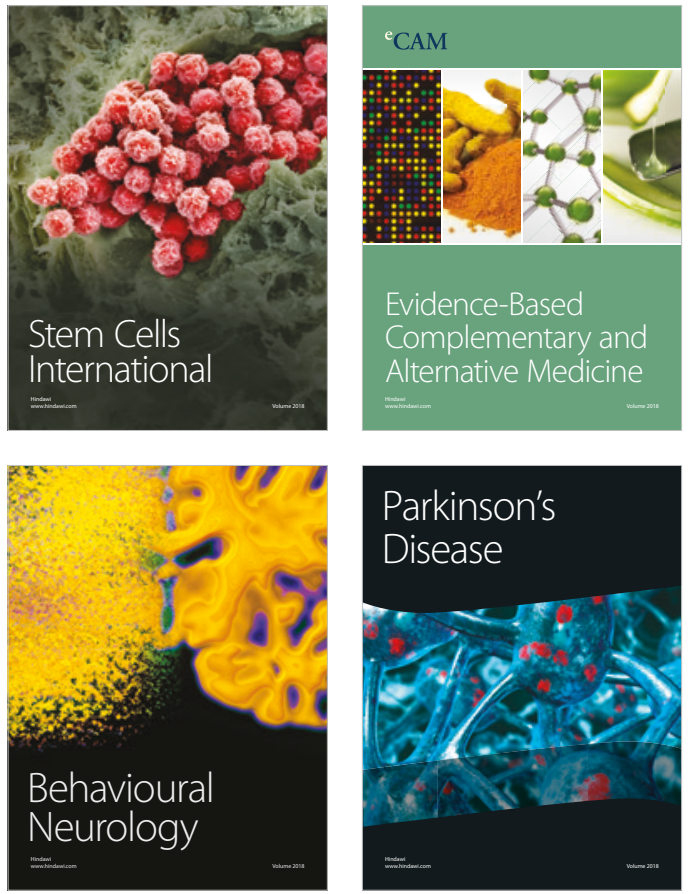

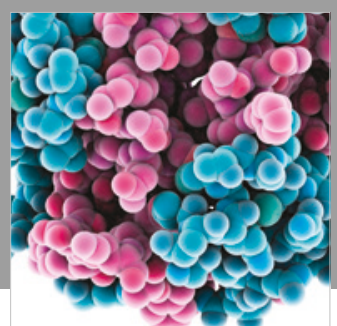

ournal of

Diabetes Research

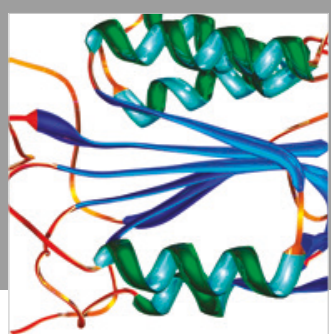

Disease Markers
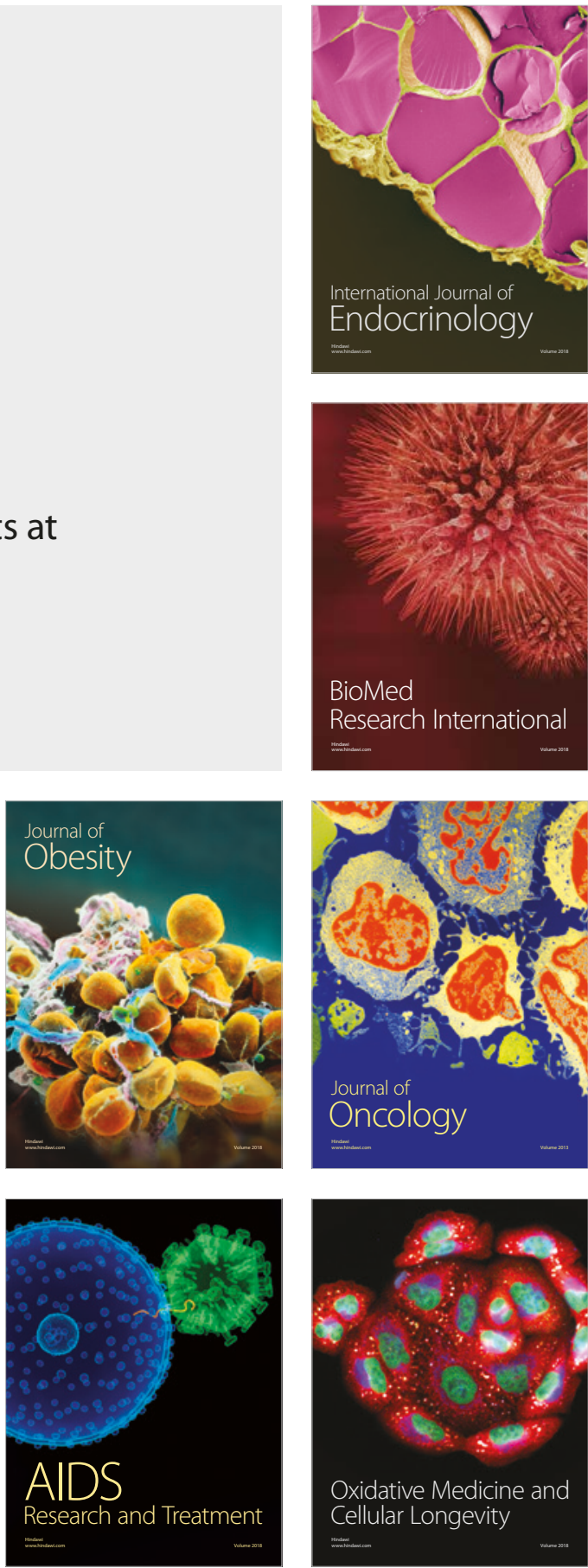